\title{
Nanocrystalline silicon carbide films for solar cells
}

\author{
S.I. Vlaskina ${ }^{1}$, G.N. Mishinova ${ }^{1}$, V.I. Vlaskin ${ }^{1,2}$, V.E. Rodionov ${ }^{1}$, G.S. Svechnikov ${ }^{3}$ \\ ${ }^{1}$ V. Lashkaryov Institute of Semiconductor Physics, NAS of Ukraine, \\ 41, prospect Nauky, 03028 Kyiv, Ukraine; e-mail: businkaa@mail.ru \\ ${ }^{2}$ Sensartech, 2540 Lobelia Dr., Oxnard, 93036 California, USA \\ ${ }^{3}$ National Technical University of Ukraine, Peremohy Ave, 37, Kiev, Ukraine
}

\begin{abstract}
Nanocrystalline silicon carbide (nc-SiC) films as protective coating and as solar cell material for a harsh environment, high temperatures, light intensities and radiation, were investigated. $p$ - and $n$-types $100-\mathrm{mm}$ silicon wafers with (100) orientation were used as substrates for $\mathrm{SiC}$ films deposition. The films were deposited using HighFrequency Plasma Enhanced Chemical Vapor Deposition (HF-PECVD) with $\mathrm{CH}_{3} \mathrm{SiCl}_{3}$ gas as a silicon and carbon source. Hydrogen supplied $\mathrm{CH}_{3} \mathrm{SiCl}_{3}$ molecules in the field of HF discharge. Deposition was carried out on a cold substrate. The power density was $12.7 \mathrm{~W} / \mathrm{cm}^{2}$. Deposition conditions were explored to prepare films with a controlled band gap and a low defect density. Formation of nc-3C-SiC films has been confirmed by the high resolution-transmission electron microscopy analysis, optical band gap values $E_{\text {Tauc, }}$ conductivity, charge carrier activation energy and Hall measurements. The efficiency of photoconductivity was calculated for evaluating the photoconductivity properties and for the correlations with technology. For $p-n$ junction creation in solar cell fabrication, the $n-$ types nc-SiC films were doped with Al. Employing Al as a doping material of nc-n-SiC, the open-circuit voltage as high as $1.43 \mathrm{~V}$ has been achieved.
\end{abstract}

Keywords: silicon carbide, nanocrystalline film, photoconductivity, solar cell.

Manuscript received 14.03.16; revised version received 12.07.16; accepted for publication 13.09.16; published online 04.10.16.

\section{Introduction}

$\mathrm{SiC}$ materials are extremely hard, very inert, and have high thermal conductivity. Their properties such as the breakdown electric field, saturated drift velocity, and impurity ionization energies are unique for different polytypes. $\beta$-SiC possesses the smallest band gap $(\sim 2.4 \mathrm{eV})$ and has the highest electron carrier mobility as compared with that of $\alpha-\mathrm{SiC}$, which makes it an important $\mathrm{SiC}$ material in the microelectronics industry [1-3]. In the tandem $\mathrm{SiC} / \mathrm{Si}$ solar cell $\beta-\mathrm{SiC}$ is an ideal top cell due to wide band gap. The $\mathrm{SiC}$ upper cell most efficiently converts the shorter wavelength light into the useful current, while the Si lower cell absorbs red and near-infrared light [1]. To prepare films with a controlled band gap and low defect density, it is necessary to investigate different structural, electro- physical and optical properties of films. Because of these excellent properties, $\mathrm{SiC}$ is a perfect material in the electronics industry, with a wide application in the areas of high-temperature, high-power, high-frequency, and optoelectronic devices [2].

This paper presents the results of investigations of nanocrystalline $\mathrm{SiC}$ films obtained using the HF-PECVD method to allow making $\mathrm{SiC}$ solar cells at low temperatures with higher open circuit voltages on large substrates.

\section{Experiment and discussion}

In this paper, the plasma-enhanced chemical-vapor deposition method (High-Frequency Plasma Enhanced Chemical Vapor Deposition HF-PECVD) was used to fabricate the nanocrystalline (nc-SiC) films. 
Methyltrichlorosilane ( $\mathrm{MTS}, \mathrm{CH}_{3} \mathrm{SiCl}_{3}$ ) was used as a source precursor, because it has an equivalent ratio of $\mathrm{Si}$ to $\mathrm{C}$ and is decomposed at a low temperature in a highfrequency (40 MHz and $13.56 \mathrm{MHz}$ ) electric field with a power density $(0.5 \ldots 12.7) \mathrm{W} / \mathrm{cm}^{2}$. nc-SiC films were deposited on the Si $(100 \mathrm{~mm})$, glass, ceramic and metal substrates at the temperatures 20 to $1000{ }^{\circ} \mathrm{C}$. Hydrogen carried out the role both of a gas carrier, which supplied $\mathrm{CH}_{3} \mathrm{SiCl}_{3}$ molecules in the field of the HF discharge, and regulator of the mixture concentration of MTS in the carrier gas. The dc bias voltage $(200 \ldots 600) \mathrm{V}$ during precipitation was supplied to substrate for increasing the drift of charged particles near the substrate surface. Deposition of $\mathrm{SiC}$ films results in solely cubic nc-SiC with average grain sizes of several tens of nanometers, as it is determined by XRD measurements. The stoichiometry of the deposited film was, therefore, continuously controlled using the Raman measurements.

The steady-state electron photoconductivity is given by:

$\sigma_{\mathrm{ph}}=e n \mu=e G \eta \mu \tau$.

Here, $n$ is the photocarrier concentration, $\eta-$ the quantum efficiency, $\mu$ and $\tau$ are the electron mobility and lifetime, respectively, $G$ is the volume generation rate of carriers.

$$
G=F(1-R)[1-\exp (-\alpha d)] / d,
$$

where $F$ is the flux of incident photons, $\alpha-$ absorption coefficient, $R$ - reflectivity, and $d$ - sample thickness.

To improve the efficiency, it is necessary to choose parameters of deposition, which will increase electron mobility. Hall measurements showed that the films deposited from $\mathrm{CH}_{3} \mathrm{SiCl}_{3}$ were characterized by sufficiently high values of the free carrier mobility and that specially undoped films possess $n$-type conductivity. The free carrier mobility in SiC-films deposited from $\mathrm{CH}_{4}+\mathrm{SiH}_{4}$ mixture was significantly lower. Fig. 1 shows the temperature dependence of the free electron concentration in films grown by using $\mathrm{CH}_{3} \mathrm{SiCl}_{3}$. Hall measurements were performed with $\mathrm{SiC}$ films deposited on $p$-Si.

The figure below shows that the concentration of electrons at room temperature for different samples varies within the small range $(2.7 \ldots 4.5)^{\cdot} 10^{17} \mathrm{~cm}^{-3}$.

The conductivity of nc-SiC is determined by the nitrogen impurity ionization. Nitrogen enters together with carrying out gas into the chamber by $\mathrm{CH}_{3} \mathrm{SiCl}_{3}$ decomposition during growth of single $\beta$-SiC crystals. Nitrogen is a shallow donor impurity with the ionization energy $\Delta E_{\mathrm{N}}=0.05 \mathrm{eV}$.

The temperature dependence of the Hall mobility shows a clear distinction between values of mobility caused by the bias voltage applied to the substrate during film growth (Fig. 2). These electrical properties of the films obtained within the temperature range $200 \ldots 600^{\circ} \mathrm{C}$ are shown in Table. Formation of nc-SiC at the temperature $200{ }^{\circ} \mathrm{C}$ is confirmed by obtained electrophysical and X-ray data [4-6].
Formation of nc-SiC films has been confirmed not only by high resolution-transmission electron microscopy analysis but by optical band gap measurements. The optical band gap values $E_{g}$ were measured using the Tauc optical method. According to Tauc's model, the optical band gap was obtained from the plot by linear extrapolation to zero.

Table. Electrical properties of SiC films.

\begin{tabular}{|l|c|c|c|c|c|c|}
\hline $\mathrm{N}$ & Structure & $\begin{array}{c}U_{\text {bias, }}, \\
\mathrm{V}\end{array}$ & $\begin{array}{c}T_{\text {subst. }} \\
{ }^{\circ} \mathrm{C}\end{array}$ & $\begin{array}{c}\mathrm{Si}_{\text {subst. }} \\
(h k l)\end{array}$ & $n\left(\mathrm{~cm}^{-3}\right)$ & $\begin{array}{c}\mu, \\
\mathrm{cm}^{2} \mathrm{~V}^{-1} \mathrm{~s}^{-1}\end{array}$ \\
\hline 1 & \multirow{3}{*}{$\begin{array}{c}\text { Poly- } \\
\text { crystalline }\end{array}$} & -600 & 920 & $(100)$ & $2.4 \cdot 10^{17}$ & 40 \\
\cline { 1 - 6 } & -600 & 920 & $(111)$ & $3.7 \cdot 10^{17}$ & 25 \\
\cline { 3 - 7 } 3 & -400 & 620 & $(111)$ & $2.4 \cdot 10^{19}$ & 12 \\
\hline 4 & -600 & 500 & $(100)$ & $1.2 \cdot 10^{17}$ & 200 \\
\hline \multirow{2}{*}{5} & $\begin{array}{c}\text { Nano- } \\
\text { crystalline }\end{array}$ & -200 & 200 & $(100)$ & $3.3 \cdot 10^{17}$ & 190 \\
\hline
\end{tabular}

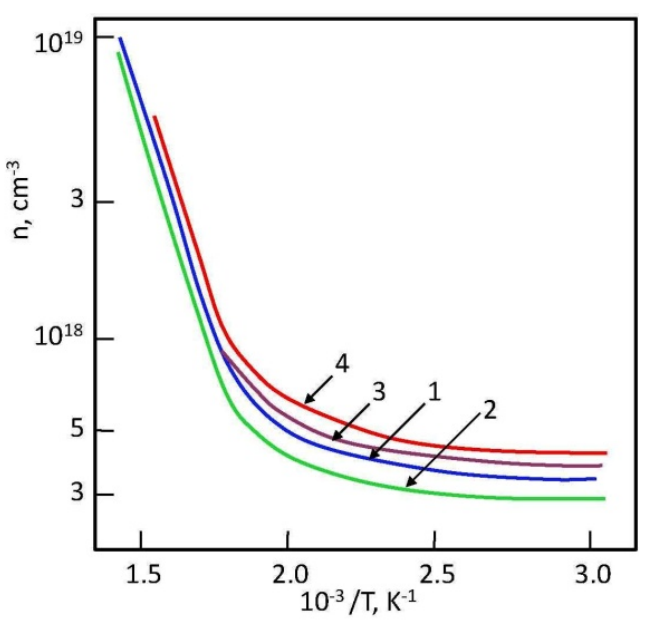

Fig. 1. Temperature dependence of the free electron concentration in films grown by using $\mathrm{CH}_{3} \mathrm{SiCl}_{3}$ : $U_{\text {bias }}=$ $+400 \mathrm{~V}(1),+200 \mathrm{~V}(2),-100 \mathrm{~V}(3),-600 \mathrm{~V}(4)$.

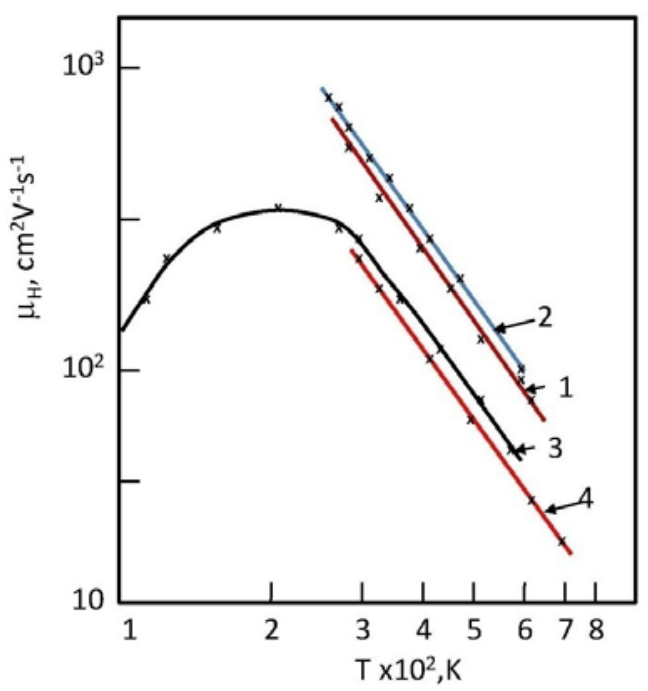

Fig. 2. Temperature dependence of the Hall mobility in $\mathrm{SiC}$ films: $U_{\text {bias }}=+400 \mathrm{~V}(1),+200 \mathrm{~V}(2),-100 \mathrm{~V}(3),-600 \mathrm{~V}(4)$. 
$(\alpha h v)^{1 / 2}=B\left(h v-E_{g}\right)$.

Here, $\alpha$ is the absorption coefficient $E_{g}$ is the optical gap, and $B$ is the constant for the given sample that characterizes the degree of order. To obtain $E_{g}$ we measured the spectral dependence of the absorption coefficient and plotted the function $(\alpha h v)^{1 / 2}$ vs $h v$. Then, extrapolation of the linear section of the curve to zero gives $E_{g}$. The thickness of nc-SiC films was $1 \mu \mathrm{m}$. The calculation of $E_{g}$ was performed as follows. The reflection from the silicon substrate and then from the film $\mathrm{SiC}$ was recorded (Fig. 3).

The absorption coefficient $\alpha$ was calculated using the formula

$I=I_{0} \exp (-\alpha d)$

where $I$ is the intensity of light reflected from the nc$\mathrm{SiC}$ film, and $I_{0}-$ intensity of light reflected from the substrate. The magnitude $d$ was taken to be $2 \mu \mathrm{m}$, since the light passed twice through the amorphous film. Fig. 4 shows that for the films deposited from $\mathrm{CH}_{4}+\mathrm{SiH}_{4}$ mixture the results of $E_{g}$ calculations are equal $E_{g}=2.55 \pm 0.05 \mathrm{eV}$. Measurements of the stoichiometric composition of this sample were carried out using an Auger spectrometer showed that the $\mathrm{Si}: \mathrm{C}$ ratio is $1: 1.5$. This result shows that the stoichiometry of $\mathrm{Si}: \mathrm{C}$ is changed from $1: 1$ to $1: 1.5$ by increasing carbon atom concentration, which leads to formation of the hexagonal polytypes of $\mathrm{SiC}$ films with a high $E_{g}$ value. Cubic $\mathrm{SiC}$ films with stoichiometric composition Si:C ratio $1: 1$ deposited from $\mathrm{CH}_{3} \mathrm{SiCl}_{3}$ showed $E_{g}=$ $2.30 \pm 0.05 \mathrm{eV}(\beta-\mathrm{SiC})$.

Raman spectra of nanocrystalline films (Fig. 5) consist of a broad band with a peak at $800 \mathrm{~cm}^{-1}$, corresponding to the maximum density of phonon states. The bright line with the frequency of $775 \mathrm{~cm}^{-1}$ is observed on the background of this broad band. The appearance of this line is also indicative of nanocrystal' occurance. Local heating the silicon substrate occurs with the bombardment of the silicon substrate with highenergy electrons, which leads to the growth of nanocrystals. Decreasing the substrate temperature down to the room one with increasing the plasma power should enhance photoconductivity.

The efficiency of photoconductivity was calculated for evaluating the photoconductivity properties and for the correlations with technology. $N_{\text {eff }}$ is a very suitable value for evaluating the photoconductivity properties of films, because there are no much difficulties in its measurement. In fact, it plays the role of the quantum photocurrent yield because $N_{\text {eff }}$ points out how many electrons participating in the photoconductivity are generated per $1 \mathrm{~s}$ by 100 photons of the full incident light focused flux on the film area $1 \mathrm{~cm}^{2}$. The parameters of photoconductivity depend on each other, and it is very difficult to measure them separately. The spectral dependence of the photocurrent (difference between the light and dark current) for $\mathrm{SiC}$ film is shown in Fig. 6.

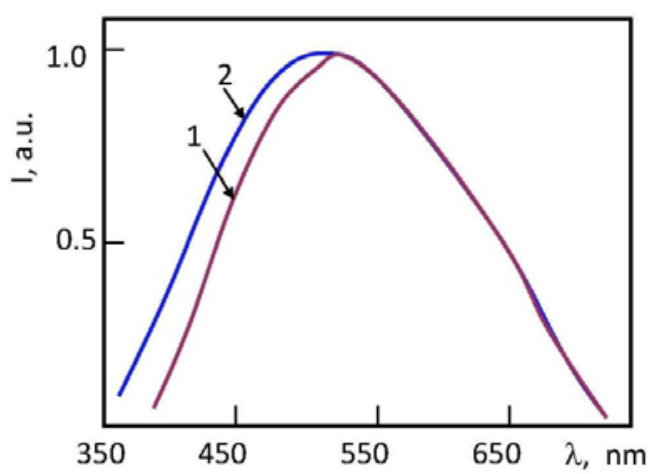

Fig. 3. Reflection from nc-SiC film (1) and from silicon substrate (2)

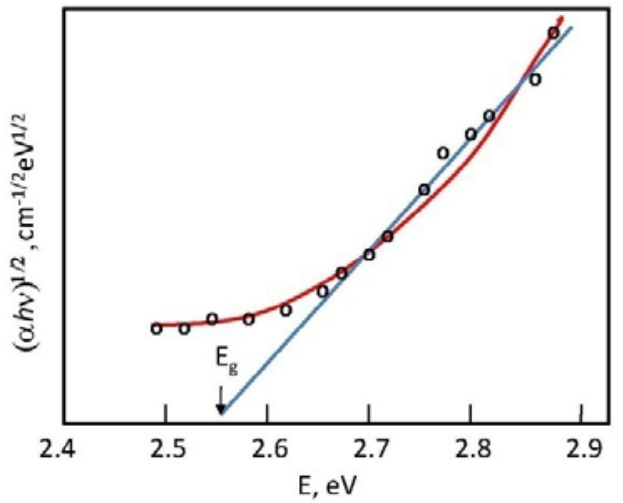

Fig. 4. Definition of $E_{g}$ according to Tauc's formula.

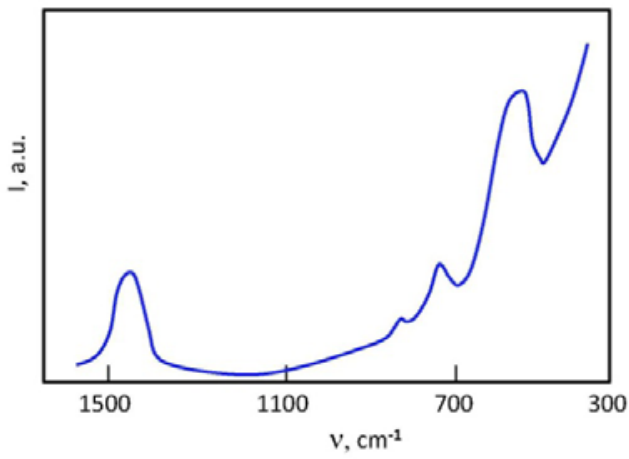

Fig. 5. Raman spectra of $\mathrm{SiC}$ film.

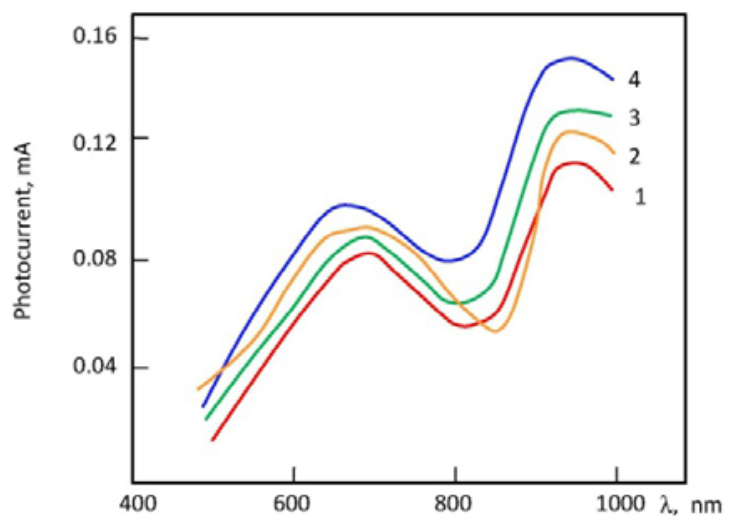

Fig. 6. The spectral dependence of photocurrent for $\mathrm{SiC}$ samples $(1,2,3,4)$. 


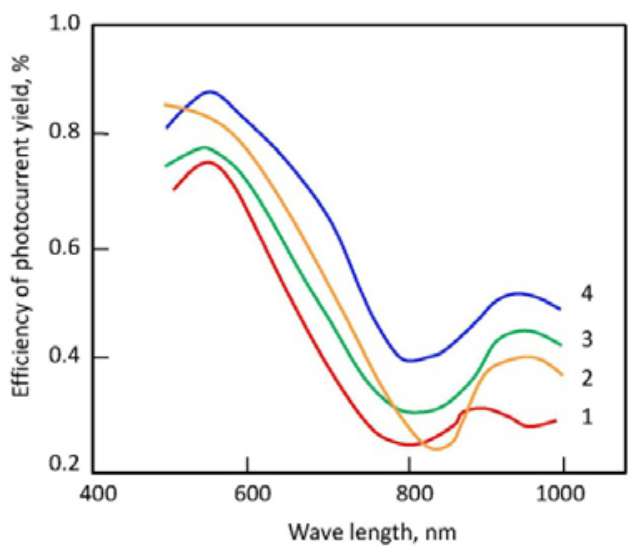

Fig. 7. Efficiency of photoelectron yield for some $\mathrm{SiC}$ samples.

To quantitatively characterize the incident light flux, it should be normalized to the density of photons per $1 \mathrm{~cm}^{2}$; then $N_{\text {eff }}$ is related to 100 photons of such a normalized light flux. In this case, expression for the photoconductivity may be rewritten as:

$\sigma_{p}=e \mu N_{\text {eff }} J / 100$,

then $N_{\text {eff }}$ can be calculated for the full spectral range (Fig. 7).
The maximum of $N_{\text {eff }}$ occurs at $\lambda=540 \mathrm{~nm}$ $(2.3 \mathrm{eV})$, which coincides with the band gap. The large halfwidth of this band is a positive peculiarity of $\mathrm{SiC}$ films, because in optoelectronic devices using these films photoelectrons are generated in the broad spectrum range. A small peak is located in $900 \ldots 1000$-nm region $(\sim 1.2 \mathrm{eV})$ and is caused by the network of defects. Thus, $N_{\text {eff }}$ measurements allowed selecting these technological parameters, which provides the highest open-circuit voltage in the manufactured solar cell.

Boron and aluminum were tried to create a $p$-layer in the nc-SiC film. More attention was paid to the development of $p-n$ junction in films obtained from $\mathrm{CH}_{3} \mathrm{SiCl}_{3}$, since those films had better optical and electro-physical parameters for solar cells. But Boron doped SiC films showed too low value $V_{o c}$. Boron-doped $\mathrm{SiC}$ films were widely used in the development of optical windows in the SiC-Si solar cell, but open-circuit voltage values for Boron-doped $\mathrm{SiC}$ film were less than $1.04 \mathrm{~V}[3]$.

The highest $V_{o c}=1.5 \mathrm{~V}$ was demonstrated by NASA Glenn Research Center, but on a small area $\left(0.48 \mathrm{~cm}^{2}\right) \quad \mathrm{SiC}$ epilayers grew at a very high temperature. This result was obtained for $n$-types $\mathrm{SiC}$ films doped with $\mathrm{Al}$. Employing $\mathrm{Al}$ as a doping material of SiC films gives $V_{o c}=1.43 \mathrm{~V}$.

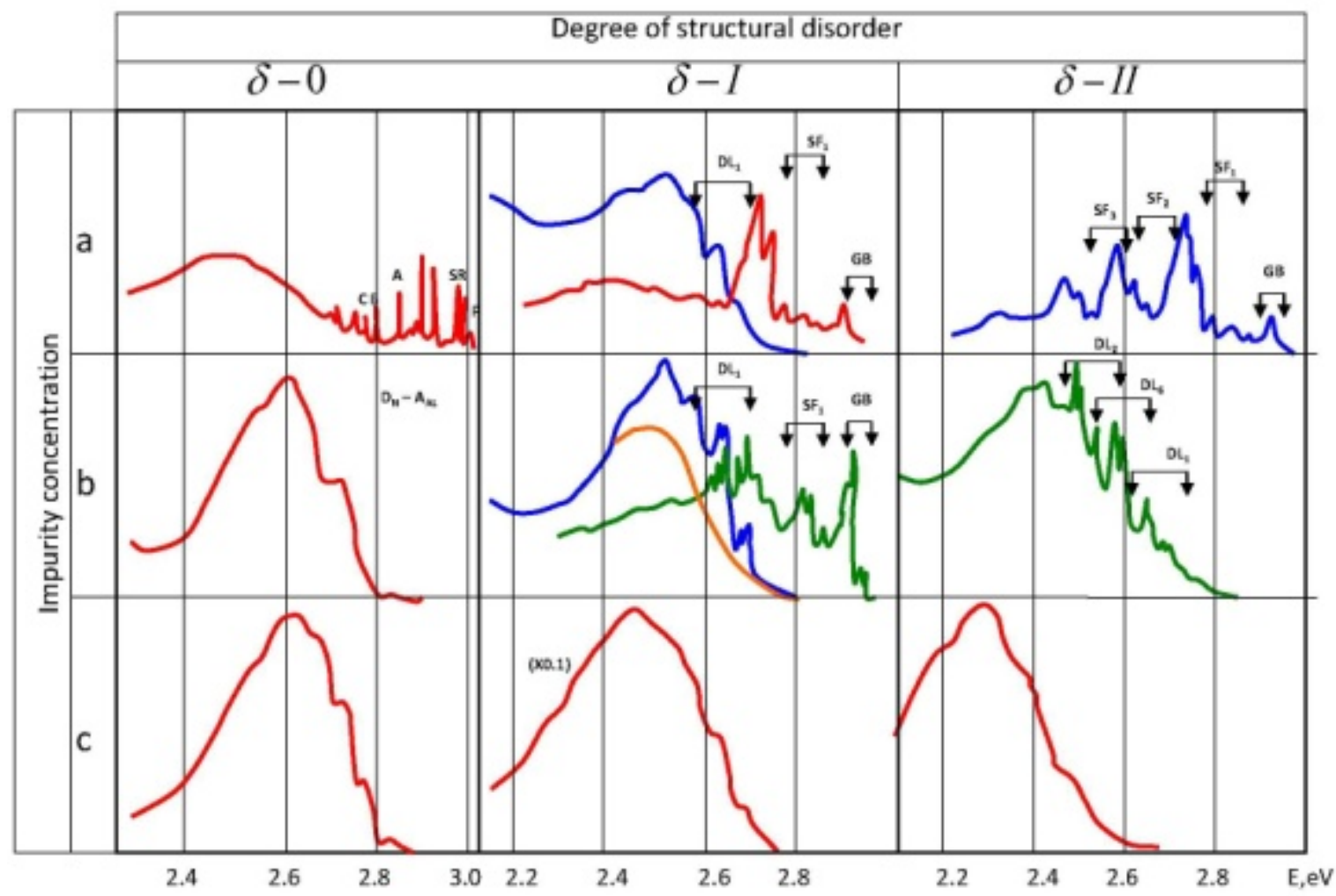

Fig. 8. Low Temperature Photoluminescence Spectra (LTPLS) according to the structural imperfection and impurity concentration in nc-SiC crystals. Zero phonon parts: $\mathrm{SF}_{1}-(2.853-2.793) \mathrm{eV}, \mathrm{DL}_{1}-(2.73-2.625) \mathrm{eV}, \mathrm{GB}-(2.95-2.89) \mathrm{eV}$. Y-coordinate: most pure films $-n \sim(1 \ldots 5)^{\cdot} 10^{16} \mathrm{~cm}^{-3}$, lightly doped films $-n \sim(5 \ldots 8) \cdot 10^{16} \mathrm{~cm}^{-3}$, samples with only DL spectra $-n \sim(2 \ldots 7)^{\cdot} 10^{17} \mathrm{~cm}^{-3}$; X-coordinate: $(\delta-0)$ - perfect nanocrystal, $(\delta-\mathrm{I})-$ the disorientation between the layers-blocks of the nanocrystal, $(\delta$-II $)$-an increased one-dimensional disorder along the hexagonal axis $\mathbf{c}$.

(C) 2016, V. Lashkaryov Institute of Semiconductor Physics, National Academy of Sciences of Ukraine 
Taking into account the results of the publications in the present study, $p-n$ junction was prepared using $\mathrm{Al}$ for doping the $\mathrm{SiC}$ films. The $\mathrm{Al}$ doped $\mathrm{SiC}$ films were heated to $600{ }^{\circ} \mathrm{C}$ despite the facts that the films were deposited on the substrate at $T=200{ }^{\circ} \mathrm{C}$ at the power of 40 to $70 \mathrm{~W}$, or on a cold substrate at the power $1000 \mathrm{~W}$ (with decomposition of $\mathrm{CH}_{3} \mathrm{SiCl}_{3}$ ). The contacts were made by sequential deposition of various metal layers at a high temperature, too. In this case, phase transformations of a cubic $\mathrm{SiC}$ to the hexagonal $\mathrm{SiC}$ were observed in nanocrystals. The ability to produce device-quality $\mathrm{SiC}$ is limited by inherent nanocrystalline defects associated with $\mathrm{SiC}$ polytypism and their associated electronic effects.

Fig. 8 shows LTPL spectra in films according to structural imperfection and the impurity concentration of nc-SiC.

The complex spectra were decomposed into similar structure-constituting spectra shifted against each other on the energy scale. These $\mathrm{SF}_{i}$ type and $\mathrm{DL}_{i}$ type spectra are indicative of nanocrystals in films, namely:

$i=1-\mathrm{SiC}-14 \mathrm{H} 1\langle 4334\rangle$,

$i=2-\mathrm{SiC}-10 \mathrm{H} 2\langle 55\rangle$,

$i=3-\mathrm{SiC}-14 \mathrm{H} 2\langle 77\rangle$,

$i=4-\mathrm{SiC}(\mathrm{N} / \mathrm{A})$, probably $-\mathrm{SiC}-28 \mathrm{H}\langle 1414\rangle$, the $\mathrm{SF}_{4}$ spectrum is difficult to determine. $\mathrm{SF}_{4}$ corresponds to the unknown polytypes with lower percentage of hexagonality (up to 7\%).

$i=5-\operatorname{SiC} 33 \mathrm{R}\langle(3332) 3\rangle$,

$i=6-\mathrm{SiC} 8 \mathrm{H}\langle 44\rangle$.

Formation of a new nano-phase is indicator of forming the corresponding motif.

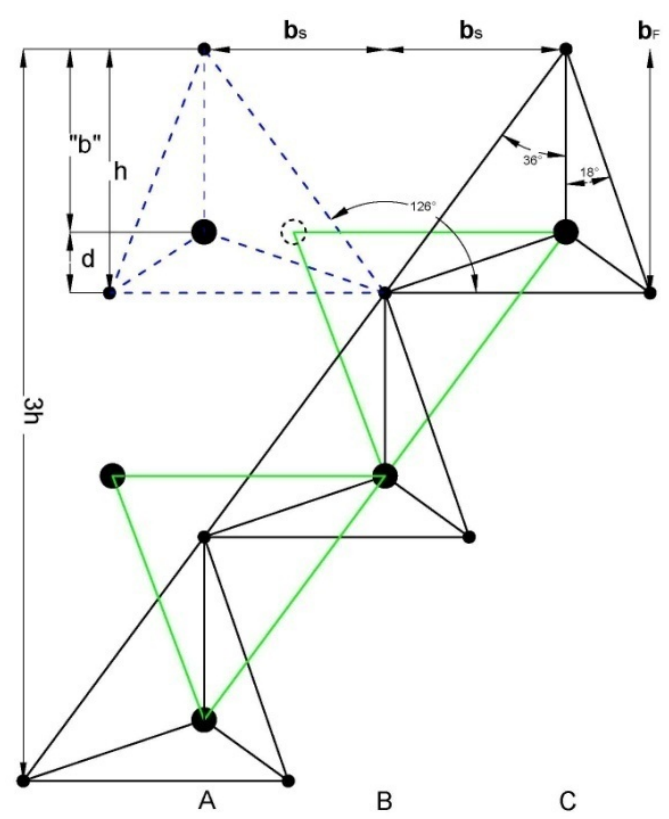

Fig. 9. The binary tetrahedral structures of nc-SiC.
Perfect nanocrystal shows a typical spectrum of nitrogen bound exciton complexes (PRS) together with the linear $\mathrm{ABC}$-spectrum related to $\mathrm{Ti}[(\delta-0)-(\mathrm{a})]$ and emission spectra of the donor-acceptor pairs. Another spectrum with zero-phonon line $(2.89 \ldots 2.94 \mathrm{eV})$ was found after identifying the SF [4] and DL spectra [5, 6]. GB spectra were observed simultaneously with the SF and DL ones (Figs. 8a and 8b) in impurity cases. Zero-phonon part of the GB spectra has a clear fine structure in the case of low films doping. GB spectrum manifests through interaction with phonons: TA-46, LA-77, TO-95, LO-104 at temperatures $(4.2-40 \mathrm{~K})$. At higher temperatures, there is a synchronous thermal decay of all the elements of the fine structure. Presumably, GB spectra display the border nanocrystalline block or interconnect boundaries. In Fig. 9, transformation of the cubic structure $(\mathrm{ABCABC})$ to the hexagonal one (ABABAB) in nc-SiC films is shown. Shockley Partial Dislocations with Burger's vector $\mathbf{b}_{\mathrm{S}}=$ $1 / 6\langle 112\rangle,\left|\mathbf{b}_{\mathrm{S}}\right|=1.788 \AA$ as well as Frank Partial Dislocation with Burger's vector $\mathbf{b}_{\mathrm{F}}=1 / 3\langle 111\rangle,\left|\mathbf{b}_{\mathrm{F}}\right|=$ $2.52 \AA$ participate in transformations.

The nc-SiC without polytype transformation (perfect crystal) were recommended for the manufacture of solar cells, because any phase transformations worsened values of short circuit current density and open circuit voltage were observed.

The short-circuit current density $J_{s c}$ and open circuit voltage $V_{o c}$ on solar cells made on our nanocrystalline films were less than those for single crystals $\mathrm{SiC}$. Namely, in the best case it was $9.78 \mathrm{~mA} / \mathrm{cm}^{2}$ and $1.43 \mathrm{~V}$, fill factor 0.71 in the case of optimized deposition conditions. $J_{S c}$ is not so good as in [1] $\left(J_{o c}=36.7 \mathrm{~mA} / \mathrm{cm}^{2}\right)$, but $V_{o c}$ is higher than $0.668 \mathrm{~V}$.

\section{Conclusion}

Films deposited using HF-PECVD with $\mathrm{CH}_{3} \mathrm{SiCl}_{3}$ gas as silicon and carbon source have better advantages than those deposited using $\mathrm{CH}_{4}$ and $\mathrm{SiH}_{4}$. Complex investigations of structural, electro-physical and optical properties allow choosing these technological parameters, which are provided by deposition films on large substrates $(100 \mathrm{~mm})$ at low temperatures (room temperature).

Deeply understanding parameters of deposition and properties of the layers allow achieving higher open circuit voltage in $\mathrm{SiC}$ solar cell. The open-circuit voltage as high as $1.43 \mathrm{~V}$ has been achieved for Al doped ncSiC. This work explores the potential possibility of producing the large area solar cells based on $\mathrm{SiC}$ for harsh environment, high temperature, light intensities and radiation applications.

\section{References}

1. Shinsuke Miyajima, Junpei Irikawa, Akira Eamada, Makoto Konagai, High-quality nanocrystalline cubic heterojunction solar cells // Appl. Phys. Lett. 97, Issue 2, 023504 (3 pages) (2010). 
2. Patent US 8,722,451 B2. Solid state energy photovoltaic device / Nathaniel R. Quick, Aravinda Kar. Date of Patent - May 13, 2014.

3. Jun Ma, Jian Ni, Jian Jun Zhang, Qun Liu, Xin Liang Chen, De Kun Zhang, Xiao Dan Zhang, Ying Zhao, High open-circuit voltage $(1.04 \mathrm{~V})$ n-i-p type thin film silicon solar cell by two phase silicon carbide intrinsic material // Solar Energy Materials and Solar Cells, 130, p. 561-566 (November 2014).

4. S.I. Vlaskina, G.N. Mishinova, V.I. Vlaskin, V.E. Rodionov, G.S. Svechnikov, 8H-, 10H-, $14 \mathrm{H}-\mathrm{SiC}$ formation in $6 \mathrm{H}-3 \mathrm{C}$ silicon carbide phase transitions // Semiconductor Physics,
Quantum Electronics \& Optoelectronics, 16(3), p. 273-279 (2013).

5. S.I. Vlaskina, G.N. Mishinova, V.I. Vlaskin, V.E. Rodionov, G.S. Svechnikov, Structure of photoluminescence DL-spectra and phase transformation in lightly doped $\mathrm{SiC}$ crystals and films // Semiconductor Physics, Quantum Electronics \& Optoelectronics, 18(2), p. 209-214 (2015).

6. S.I. Vlaskina, S.P. Kruchinin, E.Ya. Kuznetsova, V.E. Rodionov, G.N. Mishinova, G.S. Svechnikov, Nanostructure in silicon carbide crystals and films // Intern. J. Modern Phys. B, 30, No. 13, 1642019 (8 pages) (2016). 